\title{
Valerii Shevchenkol \\ COGNITIVE BASIS OF FOCAL POINTS: EVOLUTION AND CORRELATED EQUILIBRIUM EMERGENCE
}

Abstract. The paper sketches a way to connect cognitively realistic notion of relevance needed for social coordination and game-theoretic models of such coordination, in particular, that of correlated equilibrium. Such a connection would help to answer the question of how social coordination described in game theory is evolutionary and cognitively possible. The main argument put forward is to equate a signal's relevance to its information quantity - the more relevant a signal is, the more it changes probabilities of action.

Keywords: social coordination, focal point, correlated equilibrium, relevance, evolutionary cognitive science.

Для цитирования: Shevchenko V. Cognitive Basis of Focal Points: Evolution and Correlated Equilibrium Emergence // Логико-философские штудии. 2021. Т. 19, № 2. С. 131 135. DOI: 10.52119/LPHS . 2021.79 .54 .012 .

\section{Introduction}

There is a naturalistic trend in philosophy of social science addressing the problem of explanatory gaps between social and cognitive sciences (Sun (ed.) 2012; Gintis 2014; Turner 2018; Kaidesoja et al. 2019). The main tenet of this discussion is that there is empirical evidence that cognitive capacities are connected with social ones and might even ground those, but there is still no agreement on how exactly cognitive structures are connected to social ones. There are few strands within the discussion proposing different solutions.

One of the propositions is to close explanatory gaps between social and cognitive sciences using game theory and evolutionary cognitive science (Gintis 2014; Turner 2018; Guala 2020). As Turner roughly sketches, "we can think of actual societies as made up of multiple focal points which are the subject of joint attention by different overlapping groups, as the distributed rather than centralized source of multiple modes of coordination" (2018: 209). A similar notion is expressed by Guala (2020) who asks, "what kind of cognitive mechanisms facilitate coordination?" and adds that "coordination games may hold the key to understanding a wide range of important mechanisms in social ontology and social cognition generally."

The problem here is: How to connect a cognitively realistic notion of coordination and a game-theoretic one?

\footnotetext{
${ }^{1}$ Valerii Shevchenko, PhD student, HSE University. vshevchenko@hse.ru
} 
Valerii Shevchenko. Cognitive Basis of Focal Points

\section{Evolutionary origins of focal points}

Schelling's concept of "focal points" (1960) describes a psychological mechanism of equilibrium selection which lies outside of a game. One of the famous examples is the problem of choosing a place to meet without a prior agreement. Grand Central Terminal in New York City has been the recurring answer among Schelling's respondents prompted to solve the initial problem. The question is, "what makes individuals converge on this solution independently of each other?"

In game theory, there is a solution concept describing a similar situation of equilibrium emergence from outside of a game called correlated equilibrium (Aumann 1987; Vanderschraaf 1995). It describes players' decisions based on a public signal accessible to all parties, e.g. traffic lights that coordinate behavior of pedestrians as an external source of signals guiding decision-making.

Correlated equilibrium and focal points are not the same and cannot be equated, for the former is a game-theoretic solution concept and the latter is not. Instead, it is an insight needing technical accommodation from the side of game theory and ontological accommodation from the side of cognitive science.

Correlated equilibrium defines what a social convention is (Vanderschraaf 1995) - a set of beliefs formed without explicit prior agreement and with help of external signals. The concept of social convention plays a crucial role here, for it is seen as a general solution for coordination problems (Lewis 1969). The basic relationship between the concepts introduced so far is as follows: a coordination problem is generally solved by convention which is correlated equilibrium, but it is not clear what makes a certain correlated equilibrium salient, or focal. Thus, there is a gap between a focal point and correlated equilibrium - is the former a possibility condition for the latter, or are there different relations between them?

As De Freitas et al. (2019) point out, focal points make common knowledge needed for social coordination possible. It means that they serve as a source of public signals which produce common knowledge, that is one of the characteristics of conventions. However, the authors do not provide a mechanistic explanation of such a possibility condition. They highlight that individuals are sensitive even to slight differences in communication, and respond strategically in the ways predicted by the game-theoretic analysis of coordination. Thus, the mystery of the focal points holds.

From an evolutionary point of view, Skyrms (2010) argues that the problem of focal points is ruled out by evolution, for its dynamics informs equilibrium selection. Despite being plausible, this approach has its price, for it requires weakening or even dropping the assumption that individuals are rational deliberators. It roughly means that in this case individuals choosing a place to meet without prior agreement almost do not participate consciously in such a choice -instead, some evolutionary force that formed individuals' cognitive capacities "makes a decision" for them.

Though such weakening might seem controversial within game theory, it is far more 
plausible in philosophy of science. In particular, Sterelny (2012) argues that there is no only right model of rational actor in game theory - both fitness maximization as roughly sketched above and utility maximization matter, and they can be seen diachronically on an evolutionary scale, the latter after the former.

One way of saving the best of both worlds is to account for certain evolutionary formed cognitive capacities as prerequisites for social coordination which do not necessitate rational deliberation and do not discard it either. In other words, it is needed to provide a minimal evolutionary cognitive architecture which makes coordination possible and allows for rational deliberation to cover most cases of social coordination.

\section{Relevance as a possibility condition for evolutionary correlated equilibrium}

One of the more theoretically elaborated and empirically corroborated ways to think about such a condition is relevance theory put forward by Sperber \& Wilson (1986). The main idea of relevance theory is that communication, being an evolutionary formed capacity, is grounded in relevance - a characteristic of external stimuli that makes them vital to pay attention to. Animal signaling is seen as a precursor of human communication (which enables coordination) and shares with it the pragmatic relationship with the environment (Scott-Phillips 2011, 2015).

There are two principles of relevance: a cognitive one and a communicative one. The first states that human cognition is geared towards maximizing relevance, and the second states that every utterance possesses its own presumption of optimal relevance that is a characteristic of external stimuli that trades off between its processing effort and its cognitive effect (Wilson, Sperber 2002). One more important relevance-theoretic idea is that of comprehension procedure. Fundamentally, it is a cognitive mechanism making an external signal intelligible and understandable. It consists of two steps:

a. Follow a path of least effort in computing cognitive effects: test interpretive hypotheses (disambiguations, reference resolutions, implicatures, etc.) in order of accessibility.

b. Stop when your expectations of relevance are satisfied. (Wilson, Sperber 1999: 260).

Now, if we look at the possibility conditions for focal points from this angle, we might see that converging on the same place to meet without a prior agreement ceases to be a mystery, for it has a particular cognitive procedure for individuals to follow. When choosing a place to meet independently, individuals follow the steps of the comprehension procedure consecutively - by testing interpretive hypotheses that might appeal to cultural knowledge and stopping when expectations are satisfied. For example, if the individuals trying to meet are both tourists, they might consider different places and converge on the Empire State Building. 
Valerii Shevchenko. Cognitive Basis of Focal Points

The next step is to locate relevance within the framework of game theory, which is somewhat more difficult to do. One preliminary idea is to connect relevance with Skyrms' notion of the signal's information quantity (2010) - the more quantity it has, the more it changes the probabilities of action. For example, a vervet monkey alarm call changes probabilities of fellow monkeys' action to 1, according to Skyrms.

My proposal is to equate the signal's relevance and its information quantity. The more relevant an external signal is, the more it changes probabilities of action. For example, if (a mental representation) of Grand Central Terminal is optimally relevant due to some reasons, e.g. cultural, it would change the probabilities of individuals' action and they will eventually converge on the same solution to the coordination problem.

The most difficult problem in this research is to connect two drastically different academic disciplines in a non-metaphorical and conceptually precise way.

\section{References}

Aumann 1987 - Aumann R. J. Correlated equilibrium as an expression of Bayesian rationality // Econometrica: Journal of the Econometric Society. 1987. Vol. 55, no. 1. P. 1-18.

De Freitas et al. 2019 - De Freitas J., Thomas K., DeScioli P., Pinker S. Common knowledge, coordination, and strategic mentalizing in human social life // Proceedings of the National Academy of Sciences. 2019. Vol. 116, no. 28. P. 13751-13758.

Gintis 2014 - Gintis H. The Bounds of Reason: Game Theory and the Unification of the Behavioral Sciences. Revised Edition. Princeton University Press, 2014.

Guala 2020 - Guala F. Solving the Hi-Lo paradox: Equilibria, beliefs, and coordination // Minimal Cooperation and Shared Agency. Cham: Springer, 2020. P. 149-168.

Kaidesoja et al. 2019 - Kaidesoja T., Sarkia M., Hyyryläinen M. Arguments for the cognitive social sciences // Journal for the Theory of Social Behaviour. 2019. Vol. 49, no. 4. P. 480-498.

Lewis 1969 - Lewis D. Convention: A Philosophical Study. John Wiley \& Sons, 1969.

Schelling 1980 - Schelling T. The Strategy of Conflict: With a new Preface by the Author. Harvard University Press, 1980

Scott-Phillips 2011 - Scott-Phillips T. Evolutionarily stable communication and pragmatics // Language, Games, and Evolution. Berlin/Heidelberg: Springer, 2011. P. 117-133.

Scott-Phillips 2015 - Scott-Phillips T. Nonhuman primate communication, pragmatics, and the origins of language // Current Anthropology. 2015. Vol. 56, no. 1. P. 56-80.

Skyrms 2010 - Skyrms B. Signals: Evolution, learning, and information. Oxford University Press, 2010.

Sterelny 2012 - Sterelny K. From fitness to utility // Evolution and rationality: Decisions, co-operation and strategic behaviour. 2012. P. 246-273.

Sun (ed.) 2012 - Sun R. (ed.). Grounding social sciences in cognitive sciences. MIT Press, 2012 . 
Turner 2018 - Turner S. Cognitive science and the social: A primer. Routledge, 2018.

Vanderschraaf 1995 - Vanderschraaf P. Convention as correlated equilibrium // Erkenntnis. 1995. Vol. 42, no. 1. P. 65-87.

Wilson, Sperber 1999 - Wilson D., Sperber D. Relevance and relevance theory //MIT Encyclopedia of the Cognitive Sciences. 1999.

Wilson, Sperber 2002 - Wilson D., Sperber D. Relevance theory // UCL Working Papers for Linguistics 14. 2002. 